\title{
Treatment needs vary between Parkinson disease subtypes
}

T symptoms of Parkinson disease (PD), whether in the initial development or the advanced stages, can be different for every patient. This variability in what features are most prevalent has prompted the categorization of PD into subtypes, and two new studies underscore how treatment needs can differ between these groups of patients.

At the time of diagnosis, a patient's symptoms might not be severe enough to warrant intervention with a dopaminergic therapy. In a study recently published in Movement Disorders, Roberto Erro and colleagues investigated whether different features of early PD can predict when a patient will need to start treatment.

"The time to initiation of levodopa has been regarded as a reliable measure of disease progression in patients with early PD," explains Erro, "thus, we tested which baseline factors were associated with the rate of functional decline."

The investigators recruited 130 patients newly diagnosed with PD, and prospectively followed up with them for at least 2 years. During this time, Erro et al. recorded the development of motor and nonmotor symptoms, as well as the point at which patients began taking levodopa.

Just over half of the cohort (73 patients) started levodopa treatment during the study, and the investigators found no evidence to suggest that motor features in early PD were related to the need for treatment. "The overall low burden of motor symptoms in our cohort of de novo patients might explain why motor variables did not influence the statistical

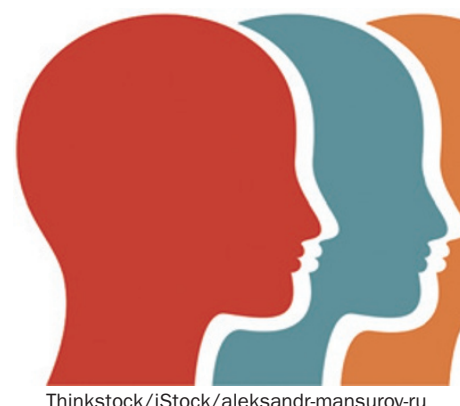

Thinkstock/iStock/aleksandr-mansurov-ru

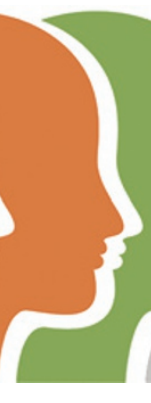

outcome," explains Paolo Barone, who supervised the study.

When the investigators looked at nonmotor symptoms, they noticed a relationship between urinary dysfunction-such as unusually frequent urination-and levodopa initiation. "The median time to treatment for patients with urinary symptoms was significantly shorter than for those without," says Erro. "Interestingly, a Cox regression model revealed urinary symptoms to be independently associated with a higher risk of needing levodopa (HR 2.13)."

The authors note that urinary symptoms were not correlated with disease duration, and, therefore, might be a true predictor of rapid progression in patients with $\mathrm{PD}$. Although further validation is essential, measurement of urinary dysfunction could help identify patients who will require early interventions. Additionally, Erro and colleagues say they intend to follow up with their cohort to see if urinary dysfunction predicts motor complications later in the disease course.

Over years-and possibly decades-of PD progression, the effectiveness and tolerability of dopaminergic therapies decreases. Deep brain stimulation (DBS) is an increasingly available treatment option for patients with advanced PD, but debate continues over what protocol is best.

In a recent issue of the Annals of Neurology, Maya Katz and co-workers describe a post hoc analysis of data collected as part of a large randomized controlled trial that compared the use of DBS in the subthalamic nucleus (STN) or the globus pallidus internus (GPi) in patients with $\mathrm{PD}$.

Using scores on the Unified PD Rating Scale, Katz's team established motoric subtypes 235 patients with PD on the basis of whether the dominant feature was tremor or postural instability and gait difficulty
(PIGD). The investigators then re-analysed the trial data to compare DBS between the two brain regions in each subgroup of patients.

Nearly $60 \%$ of the study cohort $(n=137)$ had the PIGD subtype of PD, and these patients derived significantly less benefit from DBS than did patients with tremordominant PD. The effects of DBS did not differ between the PIGD patients receiving STN or GPi stimulation. However, in patients with tremor-dominant PD, the investigators observed slightly more benefit after GPi DBS than after STN stimulation.

"Our study showed that PD motor subtype is an important variable for predicting response to DBS," concludes Katz. "The effects of each DBS target (GPi and STN) were largely equivalent across the PD motor subtypes, although there was greater improvement in gait seen after GPi DBS in the tremor-dominant group."

Because the original trial was not designed with these subgroup analyses in mind, Katz notes that additional investigations will be necessary. "Further application of our methodology to a large clinical trial initially stratified by motor subtype would help confirm our findings," she says. "Future studies could also isolate tremor-dominant patients refractory to levodopa, to see if this specific patient group has a differential response to STN versus GPi DBS.”

Together, these studies support the view that there are different motor phenotypes of PD. Further inquiry into how the differences between patients with PD affect their need for treatment could reveal strategies to manage symptoms more effectively at every stage of the disease.

\section{Alex Chase}

